\title{
Hacia una integración de la antropología urbana, del trabajo y las organizaciones. Reflexiones entre empresa y ciudad en Cali, Colombia ${ }^{1}$
}

research paper

\section{Integration of urban anthropology, work and organizations. Reflections between business and city in Cali, Colombia}

\section{Diego Hernán Varón Rojas ${ }^{2}$ Universidad del Valle, Colombia (i) https://orcid.org/0000-0001-7167-094X}

Resumen: El oficio antropológico en organizaciones empresariales y en la ciudad ocasiona un sinnúmero de retos, los cuales inciden en el ámbito académico al propiciar reflexiones en torno a la investigación y la formación académica. Este artículo incluye un itinerario de tres recorridos por una empresa y su impacto en el desarrollo de la ciudad de Cali - Colombia. Fueron tres los objetivos propuestos. Analizar las interrelaciones entre la antropología urbana y de las organizaciones empresariales y del trabajo, desde una orientación sociohistórica; desarrollar reflexiones a partir de estas relaciones y los datos cualitativos recogidos entre los años 2013 y 2017 en una empresa que presta servicios públicos, ubicada en el sur occidente colombiano; y, finalmente, comparar el comportamiento de los trabajadores antes y después de la intervención por parte de la Superintendencia de Servicios Públicos. A nivel metodológico, esta investigación se apoyó en técnicas como la revisión documental, entrevistas en profundidad y la observación participante. Algunos de los resultados permiten reconocer a las Empresas Municipales de Cali EICE ESP con una trayectoria de nueve décadas. El endeudamiento de Emcali alcanzó cifras récord en el período 1998-1999, hacia los trabajadores, entidades financieras y empresas del sector. Durante la etapa de intervención aumentaron los conflictos e hizo su aparición la desindicalización, propios de la flexibilidad laboral. La idea de que Emcali era el principal "patrimonio de los caleños" empezó a ser interrogada. A partir de los datos de investigación presentados aquí, se sostiene que los antropólogos deben aumentar la preocupación por entender los vínculos entre empresas y ciudades.

Palabras claves: antropología urbana y de las organizaciones; relaciones de conflicto; empresa de servicios públicos; Cali - Colombia.

Recibido: 7/10/2019 | Aceptado: 28/10/2020 | Disponible en línea: 3/11/2020

Como citar este artículo: Varón, D., (2020). Hacia una integración de la antropología urbana, del trabajo y las organizaciones. Reflexiones entre empresa y ciudad en Cali - Colombia. Jangwa Pana, 19 (3). Doi: https://doi.org/10.21676/16574923.3756

\footnotetext{
${ }^{1}$ Este artículo es una continuación de la investigación doctoral "El proceso de institucionalización de las Empresas Municipales de Cali - Colombia (1931 - 2016). Relaciones de poder y conflicto durante su intervención”. El proyecto fue realizado en la Universidad Eafit como estudiante del doctorado en Humanidades, en la línea de Estudios Sociales de las Organizaciones, durante los años 2012-2018, bajo la dirección del profesor Oscar Almario García.

${ }^{2}$ Correo electrónico: diegovaron@ correounivalle.edu.co.
} 
Abstract : The anthropological profession in organizations and the city creates countless challenges that impact the academic field by promoting reflections on research and academic training. This article includes an itinerary of three tours of a company and its impact on the development of Cali - Colombia. Three objectives were proposed: to analyze the interrelations between urban anthropology and those of business and work organizations from a socio-historical perspective; to Develop reflections based on these relationships and qualitative data collected between 2013 and 2017 in a company that provides public services located in Cali Colombia; and finally, to compare the behavior of workers before and after the intervention by the Superintendencia de Servicios Públicos (Governmental Controlling Unit). Methodologically, this research was supported by documentary review, in-depth interviews, and participant observation. Some of the results allow us to recognize the Empresas Municipales de Cali EICE ESP with a trajectory of nine decades. Emcali's indebtedness reached record levels in the period 1998-1999 towards workers, financial institutions, and companies in the sector. During the government's intervention to Emcali, conflicts increased, and more union labor groups appeared, typicals of labor flexibility. The idea that Emcali was the main "patrimony of Cali's people" began to be questioned. From the research data presented here, it is argued that anthropologists should increase their concern about understanding the links between companies and cities.

Keywords: Cali - Colombia; Conflict Relations; Public Services Company; Urban and Organizational Anthropology

\section{Introducción}

La inserción laboral del oficio antropológico en las organizaciones ha venido creciendo durante las últimas décadas. La publicación de investigaciones ha sido menor, en comparación con la acción participativa de creación y consolidación de organizaciones, asesorías y consultorías, y otras prácticas de los antropólogos que trabajan en las empresas. En este sentido, la producción intelectual de la antropología aplicada a contextos organizacionales y del trabajo en Colombia se encuentra aún en desarrollo.

Si la fortuna favorece al valiente, la suerte de la antropología en contextos urbanos y de ciudad ha sido diferente. Desde los años noventa se cuenta con distintos balances sobre este campo del conocimiento especializado. Pero hace falta un camino entre la madurez afortunada y la valía naciente, para nuestro contexto nacional. Este es el objetivo central de este artículo. La osadía de este viaje se apoya en trazar una ruta entre estas subdisciplinas. Se vinculan datos obtenidos durante el trabajo de campo realizado entre los años 2013 y 2017 desde una perspectiva sociohistórica, en una empresa que presta servicios públicos y está ubicada en el suroccidente colombiano, específicamente en la ciudad de Cali.

La antropología urbana como campo especializado tuvo relativa influencia de las escuelas de Chicago y Manchester (Arturo, 1993; Homobono, 2000; Guerreros, 2005). Se prestó atención a los fenómenos propios de las ciudades, pero con la diferencia de que esta está centrada en los métodos específicos del estudio de la cultura, acuñando el concepto de etnografía urbana (Mairal, 2001). La visión dicotómica entre lo urbano y lo rural, la migración del campo a la ciudad y la multiculturalidad en las ciudades aparecía ya en la obra germinal de Robert Redfield, Óscar Lewis, Richard Fox, Max Gluckman, Néstor García Canclini, y otros pioneros (Arturo, 1993; Pineda, 1993). Para el caso colombiano, se reconoce la importancia del estudio comparativo entre las clases sociales de Popayán y Querétaro (Whiteford, 1963).

Partiendo de la mirada clásica, centrada en lo arcaico, la pequeña escala y lo rural, emergieron muchas preguntas a la hora de estudiar los sujetos que migraban a las urbes. Una de estas atendía a las relaciones entre sociedades simples y las complejas (García, 2008). Es preciso

Varón, D., (2020). Hacia una integración de la antropología urbana, del trabajo y las organizaciones. Reflexiones entre empresa y ciudad en Cali - Colombia. Jangwa Pana, 19 (1). Doi: https://doi.org/10.21676/16574923.3756 
reconocer que las culturas son complejas en sí mismas, pero en la ciudad aumenta la heterogeneidad, la diversidad de sus componentes, la especialización del trabajo, las relaciones de género, los conflictos interculturales, los movimientos sociales, las expresiones colectivas y las identidades urbanas, entre otros fenómenos ampliamente estudiados.

Los debates al interior de la antropología urbana son muchos, y se generan sobre los conceptos de ciudad, urbe, espacio, territorio, urbano o urbanismo, las teorías y/o enfoques analíticos, y también sobre los métodos y las técnicas más adecuadas para su estudio. De acuerdo con las tradiciones nacionales, se diferencia el enfoque del urbanismo o las sociedades folk de los primeros trabajos, de los estudios de cultura de la pobreza o de culturas urbanas que gozan de amplia aceptación en América Latina. De otro lado, los problemas de la urbanización y colonialismo en África han tenido amplios desarrollos por parte de la antropología inglesa (Pineda, 1993), así como los debates de la cultura subalterna en Italia o la etnología urbana de Francia (Homobono, 2000).

Algunos interrogantes que se recogen de varias discusiones son ¿cuáles son los objetos/sujetos etnografiables en el contexto urbano?, ¿qué compete a la "ciudad de los antropólogos" y a "los antropólogos de las ciudades"? (De la Pradelle, 2000; Lacarrieu, 2007; Lacarrieu, Carman, y Girola, 2009). Si el mundo del siglo XXI es esencialmente urbano, ¿cómo se puede hacer realidad el derecho a la ciudad? (Prieto y Patiño, 2017). Sin importar sus vertientes y tradiciones iniciales, la antropología urbana deberá ser capaz de situar el análisis de las ciudades y de sus barrios en procesos más amplios y globales: en sociedades postindustriales. Sin lugar a duda, es preciso centrar la relación de ciudad y empresa en los movimientos protagonizados por los trabajadores. La etnografía debería estar presente en el estudio de los movimientos en defensa del "patrimonio empresarial".

En la revisión de la literatura es significativo el aumento de las perspectivas históricas y las críticas de los análisis. Como el desarrollo del capitalismo ha estado acompañado de procesos relacionados con la colonización, la migración, la industrialización y la urbanización, las orientaciones marxistas han acompañado los estudios de ciudad (Salcedo \& Zeiderman, 2008; Harvey, 2013; Martínez, 2014). Del otro lado, los debates en los estudios del trabajo y las organizaciones también son fuertes. ¿Es posible hablar de antropología industrial, de las organizaciones y de la empresa?, ¿cuáles son las distinciones entre trabajo, empleo, labor, oficio, ocupación y profesión?, ¿por qué realizar estudios organizacionales dejando por fuera el papel de los sindicatos?, ¿tienen los grupos de trabajadores preocupaciones por la ciudad? En países como Estados Unidos, Inglaterra, España, México, entre otros, hace décadas se han realizado estos balances (Esteva Fabregat, 1984; Rocca, 1998; Babor, 2009). Mientras que para el caso colombiano los puentes están por construir, los estudios entre empresa y ciudad desde una orientación antropológica cumplen alrededor de cien años.

Desde una perspectiva sociohistórica se recuerda el famoso experimento realizado en la Western Electric Company ${ }^{3}$, específicamente en la Planta de Hawthorne, a las afueras de Chicago - Estados Unidos. Entre los años 1927 y 1932 se incluyó la participación de varias

\footnotetext{
${ }^{3}$ División de la Bell Telephone System - Filial de la American Telephone and Telegraph Company.
} 
técnicas de la etnografía para la investigación empresarial y urbana. El antropólogo William Lloyd Warner ${ }^{4}$ hizo parte del grupo que diseñó estrategias en Hawthorne (Rocca, 1998; García, 2005). Las oleadas de migrantes en busca de oportunidades laborales y de ascenso social que llegaron a los Estados Unidos en el siglo XIX se cuentan como los antecedentes directos $^{5}$ (Coriat, 2005; Deegan, 2012). Gran parte de la obra de William Lloyd ha pasado desapercibida y apenas se encuentran fragmentos traducidos al español ${ }^{6}$. Este autor "no perteneció a los círculos interiores de la sociología estadounidense", y su análisis de la estratificación fue considerado como algo "demasiado cultural y no lo bastante económico para el enfoque marxista o del conflicto que se había adoptado en esa época" (Collins, 1996, p. 228).

La planta contaba con más de 30000 empleados provenientes de sesenta nacionalidades. Esta empresa, al igual que la ciudad y el país, enfrentaba conflictos interculturales. Se incluían demandas sociales generadas por los sindicatos a sus gobernantes, el aumento de la violencia organizada, los delitos de cuello blanco, etc., a las que prestaron atención los sociólogos de la Escuela de Chicago (Picó \& Serra, 2010). También, para los estudios de ciudad, el método etnográfico permitió la participación de la entrevista, la observación directa y el trabajo de campo, en la tercera y cuarta fase del experimento financiado por Fundación Rockefeller.

El préstamo metodológico de la antropología a la administración fue primero que el desarrollo conceptual con el movimiento de "cultura organizacional", generado en el campo de la gestión durante los años ochenta del siglo XX. Los ríos de tinta hallados en este viaje dan cuenta de que este tipo de publicaciones superan el campo desinteresado de los antropólogos. Si bien la administración y la gestión como ciencia aplicada ha empezado a preguntarse por los efectos irresponsables de sus prácticas en la distribución de la riqueza, la antropología desarrolló tempranamente las críticas de la empresa colonial, producto de las antropologías funcionalistas en África, Asia, América Latina y Oceanía.

El management introdujo la preocupación cultural con Ochi, Schein, Smircich, Hatch, Hofstede, Abravanel, Allaire, Firsirotu, Deal \& Kennedy, Peters \& Waterman, Aktouf, entre otros (García, 2006; Zapata y Rodríguez, 2008). Esto trajo la modelación de los valores, las creencias y los rituales de solidaridad, la motivación, el trabajo en grupo y la cohesión social, por mencionar algunos temas que incentivaron la moda empresarial que buscó entender la excelencia y la productividad. En los años recientes la cultura organizacional ha sido

\footnotetext{
${ }^{4}$ Nació el 6 de octubre de 1898 y murió en mayo 23 de 1970. Fue discípulo de Malinowski, Radcliffe-Brown y Lowie. Acababa de llegar de Australia donde realizó trabajo de campo durante tres años entre los Murngin. Pasó de estudiar el parentesco, la economía y la religión de los aborígenes australianos, para interesarse por las relaciones entre los miembros de una tribu obrera de cerca de 30.000 individuos en el corazón de la sociedad industrial.

${ }^{5}$ Los especialistas citados por Benjamin Coriat han identificado dos oleadas de migraciones. La primera, que va de 1815 a 1860, contó con un total de cinco millones de personas que desembarcaron en los Estados Unidos. La segunda transcurrió entre 1880 y 1915 con nuevas fuentes de inmigración que acabaron por alterar la composición del mercado de trabajo. La caracterización de estas oleadas incluye en la segunda mayor énfasis de los lugares de proveniencia: en 1882, el 87\% de los nuevos migrantes eran originarios de Europa del Noroeste, mientras que, en 1907, el 80,7 \% provenía de Europa del sur y del Este.

${ }^{6}$ La influencia de Warner sobre la antropología y la sociología es significativa. Ratificó el análisis de Durkheim sobre sociedades tribales y luego en industrializadas. Comprendió que la estructura social de una sociedad compleja como los Estados Unidos no se puede captar observando el sistema de estatus en comunidades separadas, por lo que investigó la movilidad social y la herencia dentro de las élites nacionales de empresarios.
}

Varón, D., (2020). Hacia una integración de la antropología urbana, del trabajo y las organizaciones. Reflexiones entre empresa y ciudad en Cali - Colombia. Jangwa Pana, 19 (1). Doi: https://doi.org/10.21676/16574923.3756 
cuestionada: ¿es una forma de consumo cultural que vincula la otredad?, ¿un instrumento de la gerencia?, ¿es una forma de control social? o quizás ¿un imperativo comercial más que un ritual de paso? (Villaveces, 1997; Dávila, 2000; Boiral, 2005; García, 2009; Trujillo, 2010).

El osado viaje se propone avanzar hacia una interrelación entre antropología urbana y de las organizaciones empresariales, desde una orientación sociohistórica. Se toma como elemento reflexivo una empresa que presta servicios públicos, ubicada en Cali - Colombia. Esta preocupación vincula datos obtenidos durante el trabajo de campo realizado entre los años 2013 y $2017^{7}$. Otro de los objetivos es comparar el comportamiento de los trabajadores antes y después de la intervención por parte de la Superintendencia de Servicios Públicos mediante el concepto de desindicalización. Algunas preguntas que se formulan en este trabajo pasan por ¿cuál es la relación entre las Empresas Municipales de Cali y la ciudad de Santiago de Cali?, ¿por qué se generaron relaciones de conflicto desde los años 90 y perduraron durante la intervención?, ¿qué aspectos propiciaron la crisis económica, política y social desarrollada en la ciudad?, ¿cómo se agudiza la crisis institucional durante la intervención realizada por un ente de control perteneciente al gobierno nacional?

Esta investigación podría arrojar luces para realizar estudios comparados en clave antropológica. Muchas empresas han sido intervenidas por la Superintendencia de Servicios Públicos por diferentes razones, como la que presenta actualmente el Caribe colombiano con Electricaribe, así como las Empresas Públicas de Medellín por su impacto social y ambiental durante la construcción de la hidroeléctrica de Hituango ${ }^{8}$. En últimas, esta actividad reflexiva intenta volver sobre algunas lecciones, quizás no aprendidas, de la principal empresa de servicios públicos del suroccidente colombiano.

\section{Materiales y métodos}

La investigación sobre la empresa Emcali y la ciudad de Cali se orientó desde una perspectiva procesual y documental. Se realizó mediante el uso del método cualitativo, apoyado en un conjunto de técnicas como fueron la observación participante, la entrevista semiestructurada y el análisis de documentos (Hammersley y Atkinson, 2001). El uso de estas técnicas permitió desarrollar los objetivos propuestos. La investigación contó con dos etapas que permitieron realizar ajustes tanto en la delimitación del problema como en la reformulación de las preguntas de reflexión.

La primera etapa implicó el uso de técnicas cualitativas para la recolección de datos, las cuales provinieron de tres fuentes: la revisión documental retomó los acuerdos del Concejo Municipal que materializaron el nacimiento y desarrollo de la empresa. La información producida por directivos y trabajadores de Emcali, junto con datos publicados en periódicos locales y nacionales, páginas web, revistas especializadas y trabajos de grado, hicieron parte

\footnotetext{
${ }^{7}$ Parte de los resultados fueron publicados en la Revista Latinoamericana de Antropología del Trabajo $\mathrm{N}^{\circ}$ 3, de 2018. Se discute en este artículo la relación trabajo y violencia, como una de las líneas que diferencia la producción intelectual del campo en Colombia, siguiendo el trabajo de Urrea y Celis (2016).

${ }^{8}$ Hidroituango es una costosa lección de ética en https://www.dinero.com/pais/articulo/las-lecciones-dehidroituango/267109 (Revisado el 10 de marzo de 2019).
}

Varón, D., (2020). Hacia una integración de la antropología urbana, del trabajo y las organizaciones. Reflexiones entre empresa y ciudad en Cali - Colombia. Jangwa Pana, 19 (1). Doi: https://doi.org/10.21676/16574923.3756 
del material hallado. Las tesis disponibles en las diversas bibliotecas universitarias de la ciudad sirvieron de soporte para conocer lo que acontecía para los años ochenta y noventa.

El uso de información proveniente de los Acuerdos del Concejo Municipal de Cali, consultorías realizadas en la empresa durante varios momentos, y periódicos de la región, resultaron significativos para el análisis de contenido presentados en la primera parte, vinculados con el nacimiento y crecimiento de Emcali. Debido a que los trabajos de grado datan del año 1980 en adelante y que la consultoría más reciente sobre temas sociohistóricos de la organización data del año 2005-2006, fue preciso realizar entrevistas y observación directa para acceder a información reciente, correspondiente a la última fase.

Las fuentes de información primarias hicieron parte de la segunda técnica de investigación, la cual consistió en la realización de entrevistas semiestructuradas. Las reuniones para el intercambio de información entre el entrevistador y el entrevistado incluyeron a seis personas, entre las que se encontraban expertos investigadores, trabajadores y directivos de la empresa. Las entrevistas se ejecutaron entre septiembre de 2013 y febrero de 2017, y fueron grabadas en formato de voz, con una duración que osciló entre 15 minutos y dos horas cuarenta minutos. Siendo coherente con las exigencias éticas, todos los entrevistados firmaron y asintieron el consentimiento informado. En algunas entrevistas también se accedió a narrativas de vida relacionadas particularmente con la crisis del despido generado después de la última toma por parte de Sintraemcali en el año 2004.

El proceso de triangulación fue necesario para validar información producto de diversos datos, razón por la cual se incluyó una tercera fuente que implicó el registro en diario de campo producto de la observación. Finalmente, se realizó observación participante en diferentes eventos organizados por el sindicato de trabajadores (Sintraemcali), bajo la discusión del Plan de Ordenamiento Territorial realizado durante el año 2015. Además, se realizó observación participante en las instalaciones de la empresa, lugar en el que se hicieron varias de las entrevistas. Producto de este ejercicio se orientó la dirección de un trabajo de grado en la Maestría en Administración de la Universidad del Valle que se enfocó en el caso de los 51 trabajadores despedidos en el año 2004. También se llevaron a cabo otras actividades como la metodología del juego de roles, desarrollada en aulas de clase con estudiantes universitarios. En estas actividades estuvieron presentes trabajadores de la empresa o los mismos estudiantes consultaron a familiares y amigos que laboran en esta organización. En resumen, se tomaron diversos apuntes que llenaron el contenido del diario de campo.

Aunque la recolección y análisis de la información ocurrió en paralelo, la segunda etapa implicó la organización de los datos, los cuales fueron recogidos y analizados a medida que avanzó el trabajo de campo. Bonilla y Rodríguez (2005) explican que la organización, análisis e interpretación de los datos en la investigación cualitativa debe realizarse a medida que se recogen. Esta fase culminó con la escritura de los apartes. Se delimitó el tiempo en dos momentos: antes de la intervención (1931 a 2000) y durante la intervención (2000 a 2013). Esta lógica se evidencia en la estructura del presente documento. 


\section{Declaración de aspectos éticos}

Los procedimientos anteriormente descritos cumplieron con los principios y normas éticas contenidos en acuerdos y normas internacionales como el convenio 169 de la OIT y las legislaciones nacionales de Colombia, donde se llevó a cabo la investigación. Se respetaron todas las formas de vida y las diversas expresiones humanas y no-humanas.

\section{Resultados}

Antes de presentar los resultados se hace necesario plantear dos delimitaciones.

1- A partir de consultorías realizadas por el Centro de Investigación de la Facultad de Ciencias Sociales y Económicas (por sus iniciales CIDSE), perteneciente a la Universidad del Valle (1994, 1994a, 1994b, 1994c, 1994d y 1994e), y de Vásquez (2001 y 2012), Camacho (2005, 2006 y 2007), y el trabajo continuado de Varela (2002, 2007 y 2008), se han identificado diversos períodos y características más relevantes de las Empresas Municipales de Cali. Este trabajo propone una periodización sociohistórica distinta a la planteada en los trabajos anteriores. No se presenta por décadas, como la establece la obra de Miguel Camacho apoyado en el trabajo pionero de Edgar Vásquez, o por los servicios prestados por Emcali, como lo plantean los resultados del CIDSE. Tampoco está en clave de política pública, como lo ha estudiado Edgar Varela, sino que se plantea en dos momentos con un período de transición.

2- Debido a la temporalidad tan extensa, se genera un énfasis de la relación empresa-ciudad, y el aporte más significativo de esta relación se concentra entre los años cuarenta y los noventa. Los casi 90 años de existencia son divididos en el período comprendido entre los años 1931 y 1990, seguido de un período de transición generado por cambios en el contexto de la empresa, y el tercer momento incluye la materialización de la intervención que duró 13 años (2000 a 2013), así como un breve período de readaptación que llega hasta el momento actual. Es tan significativa esta relación sociohistórica entre Emcali y Cali, que varios medios de comunicación informaron a su audiencia, en junio de 2013, que la empresa había sido "devuelta a la ciudad". Antes de presentar este final, feliz para algunos y terrible para otros, sugiero empezar el recorrido desde sus inicios.

\section{Las Empresas Municipales de Cali entre los años 1931 - 1990}

En las primeras décadas del siglo XX, Santiago de Cali vivió el tránsito hacia su modernización. Este recorrido estuvo marcado, entre otros aspectos, por la navegación comercial en el río Cauca, el transporte por el ferrocarril del Pacífico, el desarrollo manufacturero, la consolidación de diversas áreas de la alcaldía para prestar servicios públicos y, en 1931, la aparición de las Empresas Municipales (Vásquez, 2012). Entre 1910 y 1930, la ciudad estaba ceñida a la colina de San Antonio y su expansión se proyectaba en todos los sentidos, buscando salidas a otras regiones importantes en términos comerciales, pero conservando siempre una forma rectangular (Vásquez, 2001). En las décadas siguientes, la ciudad atravesó la senda del crecimiento poblacional y empresarial, por lo que se requirió que las empresas de servicios públicos se desarrollaran para soportar las demandas requeridas. 
Las obras de servicios públicos realizadas en las primeras décadas del siglo $\mathrm{XX}$ fueron construidas a partir de interacciones humanas, generando ventajas para la urbe recién elegida como capital del Departamento del Valle del Cauca, creada en 1910 (Almario, 2013). En efecto, las comodidades empezaron a aparecer desde ese año, cuando fue instalada la primera planta eléctrica privada por iniciativa de Henry Eder. Entre 1914 y 1918 se construyó el primer acueducto que reemplazó la red de pilas y las acequias que surtían de agua a los sectores populares. Este acueducto se construyó en la colina de San Antonio y a partir de él se constituyeron las Empresas Municipales (CIDSE, 1994a; 1994b).

Con la integración de una junta administradora para el servicio de alcantarillado, la administración de las plazas de mercado y otros servicios prestados por la alcaldía de Cali, en 1931 se crearon las Empresas Municipales. La ciudad empezó una fase de crecimiento poblacional y empresarial, razón por la cual se requería aumentar la cobertura de servicios. Las empresas privadas de energía y teléfonos en 1944 no lograban satisfacer la demanda de los usuarios, así que estos servicios prestados por empresas privadas fueron anexados mediante un proceso de nacionalización, fenómeno que se presentaba en el país por esa época (CIDSE, 1994c; 1994d). Mediante los acuerdos $\mathrm{N}^{\circ} 36$ y 50 de 1961, se le permitió a las Empresas Municipales de Cali (en adelante Emcali) adquirir autonomía para recurrir a préstamos internacionales con el fin de aumentar la construcción de infraestructura necesaria para mejorar los servicios.

$\mathrm{Si}$ bien las Empresas Municipales fueron creadas como parte del proceso social experimentado en los años 30, entre los 60 y los 90 del siglo XX se desarrolló un período de expansión importante que les permitió consolidarse como una de las instituciones más representativas de la ciudad. El modelo de gestión utilizado en la prestación de Servicios Públicos Domiciliarios (SPD), implementado a partir de los años 60, contribuiría a dignificar la vida de los habitantes de Cali y la región. Durante estos años se vivió una etapa de crecimiento que se explica, en parte, por el ambiente de certidumbre presentado en los valores de la gente, las disposiciones jurídicas y políticas, la estabilidad económica, el auge de las empresas y la migración que esto atrajo, la activación de la construcción y el crecimiento de la ciudad (Vásquez, 1990; Camacho, 2005; Varela, 2008; Varón, 2018a).

La relativa estabilidad generada en la prestación de SPD también fue posible al prevalecer un modelo político y económico diseñado y ejecutado por el Estado (Vélez, 2013). De esa forma se le permitió prestar servicios públicos como parte de la función social enmarcada hacia el bienestar. El Estado local depositó en las Empresas Municipales su más grande aliado, la responsabilidad de atender las demandas generadas por el crecimiento de la ciudad, aumentando la cobertura, ampliando redes, mejorando la sanidad y las condiciones de vida de los habitantes de Cali (Vásquez, 2001). Lo anterior, la facultó para ocupar un lugar destacado frente a los procesos de modernización, desarrollados particularmente en Colombia desde la segunda mitad del siglo XX.

A comienzos de los años 60, se estimó la población de Cali en 465000 habitantes, de los cuales 347000 usuarios contaban con agua potable. De esa forma, 118000 personas carecían

Varón, D., (2020). Hacia una integración de la antropología urbana, del trabajo y las organizaciones. Reflexiones entre empresa y ciudad en Cali - Colombia. Jangwa Pana, 19 (1). Doi: https://doi.org/10.21676/16574923.3756 
del servicio o contaban con él, pero de forma deficiente. Por esta razón, Emcali solicitó formalmente un préstamo al BID en marzo de 1961 y en octubre del mismo año se le concedió el empréstito BID9TF por US\$ 2454000 provenientes del Fondo Fiduciario de Progreso Social $^{9}$. Como el BID se había constituido el 30 de diciembre de 1959, el empréstito "concedido a Emcali fue uno de los primeros adjudicados por dicha institución bancaria" (Emcali, 1961, folio 4). Estos dineros fueron invertidos en el ensanche del sistema de agua potable en los 60, que consistió en la compra y colocación de tuberías matrices que alimentaban las nuevas áreas industriales y zonas residenciales, compra y colocación de redes de distribución para los barrios populares y la construcción de cuatro tanques de almacenamiento indispensables para regular el suministro de agua en la ciudad (CIDSE, 1994a; Vásquez, 2001; Camacho, 2006).

Para blindar la empresa de los conflictos bipartidistas constantes durante el curso de la historia de Colombia, en el artículo 17 se definió a Emcali como un organismo técnico y administrativo "en el sentido de que no será regido en ninguna de sus actividades ni sesiones con criterio de política partidista. Será dirigido siempre con un riguroso criterio de eficacia administrativa", empleando modernos sistemas técnicos para garantizar el funcionamiento "regular, permanente y económico de los servicios públicos" (Gaceta municipal № 747, mayo de 1961 a enero de 1962, p. 46), fomentando el progreso de Cali. Las distinciones políticas de los partidos tradicionales se hicieron presentes en los acuerdos que desarrollaban la composición de la Junta Directiva, específicamente entre los artículos 23 al 26, buscando que sus seis integrantes principales tuvieran "paridad política" con representación de las dos colectividades tradicionales.

Irving Webber y Selwyn Hollingsworth (1975) realizaron un trabajo en Santiago de Cali que indagó por la articulación entre los valores sociales y los procesos de desarrollo instrumental. En este trabajo ya se reconocía el posicionamiento que las Empresas Municipales de Cali tenían entre los líderes de la región. Más allá de resaltar la importancia de los lineamientos teóricos y metodológicos que se fundamentó en la propuesta de Florence Kluckhohn con una medición cuantitativa, se resalta la ubicación de Emcali como una "entidad cuasigubernamental". La investigación consultó a un total de 59 dirigentes de Cali, y al conversar con estos "informantes bien calificados" se concluyó "que las Empresas Municipales (...) y la Unidad de Acción Vallecaucana, creada para promover el desarrollo del departamento, eran las dos entidades cuasigubernamentales de mayor importancia" en la región (Webber y Hollingsworth, 1975, pp. 155-156). La opinión de los expertos locales registrados en el estudio añade información para afirmar que, para los años 60, las Empresas Municipales de Cali ya eran una entidad posicionada en la mente de sus habitantes. Se percibía como una institución que aportaba significativamente a la promoción de valores cívicos y a las dinámicas de modernización de la ciudad.

\footnotetext{
${ }^{9}$ Para estos años se iniciaron los programas de cooperación internacional conocidos como la "Alianza para el Progreso", patrocinado por el gobierno de Kennedy e instaurado a partir de la "Carta de Punta del Este" (Palacios, 1995). El objetivo de estos programas era contrarrestar las ideas comunistas que rondaban en el periodo de la Guerra Fría, y que estuvieron alineados con las políticas de Estado implementadas por gobiernos, como el de Alberto Lleras Camargo (1958-1962). Para comprender su impacto en entidades que financiaron proyectos con recursos por Cooperación Internacional en Cali, se puede estudiar el caso de la Fundación para la Educación Superior (FES) creado hacia 1964 (Crespo, 2011).
}

Varón, D., (2020). Hacia una integración de la antropología urbana, del trabajo y las organizaciones. Reflexiones entre empresa y ciudad en Cali - Colombia. Jangwa Pana, 19 (1). Doi: https://doi.org/10.21676/16574923.3756 
¿Por qué las Empresas Municipales de Cali fueron incluidas como una institución cuasigubernamental? Son variados los argumentos que están relacionadas con el compromiso de la ciudad, la participación en los primeros planes de desarrollo y la promoción de valores orientados a generar tejido social. Directivos, trabajadores de la empresa y ciudadanos fueron responsables de esta construcción social que se consolidaba con el paso de las décadas en el mantenimiento y la administración de los servicios públicos de la ciudad y municipios aledaños. Algunos miembros de la junta directiva y gerentes también hacían parte de las élites tradicionales (Sáenz, 2010), de forma que el modelo de empresa diseñado para Emcali no permaneció distante del proyecto de ciudad planificado en los años 60 (Martínez y Buitrago, 2011).

Veinte años después, la sultana del Valle se consolidaba como la tercera ciudad más poblada del país, "con estructuras modernas de población y una rica diversidad étnica-racial", constituyéndose al mismo tiempo en una "ciudad mestiza y negra" (Urrea, 2012). Las gentes negras han nutrido históricamente la mano de obra de las empresas modernas ubicadas en la región. La existencia de empresas modernas con mercados de trabajo internos del período de sustitución de importaciones fue dominante en la región del Valle del Cauca (1950-1980), en particular en el cinturón Cali-Yumbo. También en empresas del sector público, como Emcali, fue favorable la incorporación de trabajadores negros y mulatos (Urrea, 2011, p. 37). La mayor parte de estas "capas sociales negras" fueron conformadas por caleños e inmigrantes de regiones cercanas, como el norte del Cauca y sur del Valle, incluida la ciudad de Buenaventura.

Finalizando los años 80 se generaron dos cambios jurídicos relevantes en el devenir urbano y político de la nación, cuya incidencia local no tardó en añadirle complejidad a las Empresas Municipales de Cali: la distribución del espacio urbano en comunas y las Juntas Administradoras Locales (JAL) para su administración, que incidirían en la concepción del territorio para prestar los Servicios Públicos Domiciliarios (SPD), así como la ubicación de oficinas de recaudo para descentralizar mediante los Centros de Atención Local Inmediata (CALI). Esto, sumado a la elección popular de alcaldes que participaría en la elección del gerente general, así como en los directivos que son asignados según la "discrecionalidad" de este importante cargo, empezó a transformar la gestión político-administrativa de la empresa. En resumen, durante los años 1931 y 1980 las Empresas Municipales de Cali adquirieron su madurez.

\section{Un período de transición: los difíciles años 90}

Al generarse cambios legislativos para adaptarse a las transformaciones políticas y económicas del contexto internacional bajo la lógica de la apertura económica, se presentaron desafíos que pusieron en aprietos a las Empresas Municipales de Cali. En los años noventa, con el deseo de aprovechar la independencia generada con el nuevo modelo gerencial, se incorporaron muchos proyectos, a fin de consolidarse como una gran empresa con inversión privada. El rostro de este crecimiento estuvo acompañado con el crecimiento de las deudas, las prácticas de corrupción y clientelismo, y las disputas entre distintos bandos por el poder. El contexto de ciudad no era distinto al de la empresa. El modelo de civismo junto con las acciones de planeación urbana por el que Cali había sobresalido en los años 80, rápidamente 
se había transformado, emergiendo altos niveles de violencia, desempleo, pobreza y marginalidad.

La Superintendencia de Servicios Públicos Domiciliarios (SSPD) fue creada con la Ley 142 de 1994, para controlar el desempeño de las empresas y garantizar el buen servicio a los ciudadanos. Hacia el año 2000, más de veinte empresas públicas prestadoras de servicios públicos domiciliarios habían sido intervenidas ${ }^{10}$. Trece años después, Empresas Municipales de Cali EICE ESP fue una de las pocas que continuó siendo pública. En el año 2013, Emcali fue retornada a la administración por parte del Municipio de Santiago de Cali. ¿En qué momento se generó el colapso de estas empresas? y ¿por qué colapsaron?

La crisis energética vivida en Colombia en los años 90 obligó a pensar en nuevas alternativas que buscaban combatir el fantasma del nuevo racionamiento. Para la ciudad de Cali, la generación de energía había sido un proyecto postergado desde los años 40. Las innovaciones tecnológicas a partir de las termoeléctricas fueron presentadas como la panacea. Apostarle a la construcción de térmicas en una región donde el gas es escaso, terminó siendo una decisión gerencial costosa e insuficiente, lo que significó para Emcali un aprendizaje forzado sobre las malas inversiones representadas en Termoemcali, Termopacífico y Termovalle. Las evaluaciones fueron realizadas durante los primeros años de intervención por parte de la SSPD. Parte del endeudamiento excesivo no solo se debió al asumir responsabilidades con el medio ambiente al construir la Planta de Tratamiento de Aguas Residuales PTAR, sino que la crisis del sector energético terminaría golpeando a la empresa patrimonio de Cali y la región.

Durante los años 1991 y 2000 se generaron cinco factores que desencadenaron la crisis institucional de Emcali (Varón, 2018a). En primer lugar, se reconoce la crisis económica, político-institucional y fiscal vivida en la ciudad, así como el recrudecimiento de la violencia física generada por distintos actores, entre ellos los integrantes del cartel de Cali, las guerrillas y la delincuencia organizada (Guzmán, 1993, 2012). El segundo factor incluye a los directivos de las Empresas Municipales de Cali y líderes políticos de la región que buscaron adaptarse anticipadamente a los cambios regulatorios, razón por la cual auspiciaron la celebración de eventos con invitados de países suramericanos que ya habían vivido este proceso. También se contrataron diversos consultores nacionales e internacionales para que diseñaran el modelo empresarial, incluso antes de conocerse las modificaciones generadas con la Ley 142 de 1994 (Camacho, 2006; Varela, 2008). Las propuestas realizadas por los consultores generaron en mayor o menor medida aceptación por distintos grupos sociales, pero también el rechazo por los directivos de la empresa y los trabajadores vinculados al sindicato. Se abría la posibilidad de inversionistas privados para contrarrestar la corrupción que ya hacía su aparición en el escenario público (González, 2003; Martínez \& Ramírez, 2005).

\footnotetext{
${ }^{10}$ http://www.eltiempo.com/archivo/documento/MAM-1220558 (Consultado el 10 de enero de 2018).
} 
El tercer factor incluye los escándalos de proporciones nacionales generados por la vinculación de dineros provenientes del narcotráfico para financiar campañas presidenciales durante el gobierno de Ernesto Samper (1994-1998), que no tardaron en tener un impacto sobre la economía regional y específicamente en el desempeño de su Empresa Municipal. Algunos directivos y trabajadores fueron llamados a indagatoria por vínculos con el cartel de Cali, especialmente del área de telecomunicaciones (Entrevista 1, noviembre 14 de 2016; Rempel, 2011; Varón, 2018b), aspecto que afectó la rotación de gerentes y directivos de mandos medios. Las denuncias de corrupción se volvieron constantes (Varela, 2002), así como las muertes violentas de trabajadores de Emcali (Celis, 2004). La legitimidad de la empresa se vio duramente cuestionada.

En cuarto lugar, es representativo de este período el debate político suscitado entre la alcaldía, el Concejo Municipal, la gerencia de la empresa, los trabajadores organizados principalmente en Sintraemcali ${ }^{11}$ y Asiemcali, las élites regionales, los medios de comunicación, los líderes de las Juntas de Acción Comunal y otros grupos pertenecientes a la comunidad. El cambio del modelo para las Empresas Municipales de Cali desató la disonancia y la conmoción, pero finalmente fue aprobado el Acuerdo 14, de diciembre 26 de 1996, que transformaría a Emcali en un holding compuesto por un centro corporativo y cuatro empresas prestadoras de SPD (Entrevista 2, enero 27 de 2014; Ochoa, 2010). Este acuerdo tendría una corta vida porque sería derogado en enero de 1999, bajo el mismo escenario de inconformismo por parte de diversos sectores, principalmente del sector empresarial que esperaba la privatización.

Finalmente, se relacionan las grandes inversiones que buscaban expandir los negocios de Emcali. Durante el breve lapso de autonomía a la que accedieron las empresas producto del holding en 1996, se materializaron varios proyectos que venían siendo planeados desde hacía varios años. Se buscaba devolverle la potabilidad de las aguas servidas del río Cauca con la construcción de la PTAR. De otro lado, los líderes de la ciudad y la empresa deseaban producir energía aprovechando la tecnología de las térmicas. Los dirigentes de la ciudad y de Emcali buscaron estar a la vanguardia en el ámbito tecnológico y económico, también frente a las transformaciones socio-jurídicas que se veían llegar con el neoliberalismo de los años 90 (Camacho, 2007; Varela, 2007).

Para el año de 1999 se habían intervenido varias compañías: la Empresa de Energía Eléctrica de Arauca (Enelar), la Empresa de Servicios Públicos del Oriente (Espo), Centrales Eléctricas del Cauca (Cedelca), Empresa Industrial y Comercial de Cúcuta, Empresas Públicas de Caucasia y la Electrificadora de Chocó. En marzo de 2000 se buscaban salidas como la venta, la concesión o la liquidación de estas empresas. La SSPD entró en proceso de liquidación de

\footnotetext{
${ }^{11}$ Sintraemcali nació pocos años después de haber sido creada la empresa, el 29 de mayo de 1937 (Celis, 2004). Los trabajadores organizados no fueron una fuente de oposición a las políticas y reglamentos de la empresa, aun cuando en los años 30 se vivieron agitaciones por las condiciones económicas. En los 40 las movilizaciones sociales permitieron la estatización de servicios de energía y teléfonos. Los trabajadores de las Empresas Municipales se plegaron a los valores instaurados por los directivos, a pesar de que el historial huelguístico en Cali era grande, generado en empresas del sector ferroviario, de textiles como La Garantía, en la Cervecería Colombia, pero también de choferes, mineros y vendedores de la plaza de mercado (Serrano, 2012). Se ubicó en la casa de Alcides Arcos en el barrio Alameda. Su primer presidente fue Jorge Navia Paz y pronto ingresaron a la Confederación Sindical de Colombia. Un año después se fundó el primer fondo de empleados y pronto la cooperativa de trabajadores de la empresa sería conocida como Cootraemcali (Camacho, 2005).
}

Varón, D., (2020). Hacia una integración de la antropología urbana, del trabajo y las organizaciones. Reflexiones entre empresa y ciudad en Cali - Colombia. Jangwa Pana, 19 (1). Doi: https://doi.org/10.21676/16574923.3756 
las electrificadoras de la Guajira, Magdalena, Atlántico, Bolívar, Sucre, Córdoba, Cesar y Magangué. Las funciones de comercialización fueron asumidas por las electrificadoras de Electricosta y Electrocaribe.

\section{Conflictos por Emcali entre los años 2000 y 2013}

De la incertidumbre con la que empezó el siglo XXI ni las Empresas Municipales de Cali se habían salvado. En parte, la falta de certeza se debía a la alta dependencia tecnológica que ahora tienen y las empresas, pero en el caso de Emcali era más que tecnología. Los rumores de una posible intervención por parte del gobierno nacional se propagaron por toda la ciudad y sus alrededores. La crisis financiera era insalvable en el corto plazo y eso lanzaba por la borda los modelos de gestión de décadas pasadas (Zuluaga e Idrobo, 2000; Cardona, 2002). Sin embargo, la situación financiera no era sino una de las aristas. En juego se encontraba la pujanza de las gentes de la región, la construcción social de ciudad, la reputación que fue el orgullo de los caleños y la capacidad emprendedora de sus habitantes. Emcali realmente era sentida como patrimonio de la comunidad, y la clase empresarial fundamentaba su compromiso con acciones direccionadas hacia "el buen gobierno", presente en valores de civismo, participación, solidaridad.

Se resume en cuatro aspectos la agudización de la crisis: las condiciones económicas y políticas cambiantes del país exigieron que los recursos prometidos para la construcción de la PTAR se diluyeran, de forma que el documento Conpes, donde había quedado explicitada la promesa, misteriosamente había desaparecido. A las reglas de juego cambiantes se le sumó el cambio de valores basados en la corrupción. Escándalos como el de Enrom a nivel global fueron explicados por la volatilidad financiera y la levedad normativa asumidos con la globalización. A la crisis financiera regional se le habían sumado dineros del narcotráfico y la entrada de la apertura económica, aspectos contextuales que afectaron las finanzas de los usuarios, el municipio de Cali y por supuesto a Emcali, que había dejado de cumplir sus obligaciones con los proveedores razón de la intervención. Los contratos firmados con empresas extranjeras para la realización de onerosas obras realizadas por Emcali fueron denunciados como espacios de corrupción (Martínez y Ramírez, 2005; Vélez, 2013). Escándalos planteados desde el "periodismo investigativo" terminaron afectando la poca legitimidad que le quedaba a la empresa durante los años de intervención.

El segundo elemento vinculado con la agudización de la pérdida de institucionalidad de Emcali tiene que ver con la entrada en vigencia de la libre competencia y el fantasma de la privatización, que causó desestabilidad sobre la empresa. La privatización ha sido considerada por muchos analistas como un "proceso que va rumbo a la desinstitucionalización de la institución” (Pesqueux, 2009, p. 8). Desde la racionalidad económica liberal, una empresa pública tiene el imperativo de producir inversión social. Ante la "ineficiencia operativa", además de ser intervenida, Emcali debería ser liquidada. Las presiones del gobierno central, especialmente desde la SSPD, expresaron en reiteradas oportunidades la necesidad de privatizar. Los recursos provenientes de Emcali, generados para la inversión social de la ciudad, habían desaparecido. 
Durante los tres primeros años de intervención, la falta de acciones concretas para salir de la crisis financiera incidió en la agudización de la inercia institucional. Esta se generalizó por las continuas disputas de poder. El conflicto consecuente entre diversos actores ubicados en escalas diversas que mantuvieron la lógica de la oposición, presentó diversas estrategias siendo las más significativas las movilizaciones y tomas, las denuncias públicas y las alianzas internacionales. Retomar las riendas de la institucionalidad no fue tarea fácil. La rotación continua de gerentes y trabajadores de confianza para altos cargos configuró una arista del problema ante la falta de estabilidad normativa. Esto fue producto de la novedad institucional representada por la reciente SSP. Estas disputas caracterizaron el fenómeno mismo de intervención.

Una parte de las fuerzas regionales presionó al gobierno central para lograr mayor inversión social. Bajo la lógica del Estado de Bienestar ${ }^{12}$ se buscaba que la deuda por más de un billón de pesos que Emcali le adeudaba a la nación fuera perdonada. Pero la lógica reinante en el siglo XX se direccionaba hacia el libre mercado, de forma que la reducción del Estado se redujo a generar "ciertas regulaciones". El clamor fue constante durante la intervención. Se buscaba una medida salvadora unida al clamor ciudadano de "Emcali como patrimonio de los caleños". El mecanismo de salvación consistía en que el ejecutivo asumiera la responsabilidad pecuniaria de la PTAR, dada la relevancia nacional que este proyecto había representado para el país. Despojada de toda institucionalidad durante la intervención, Emcali pasó a ser administrada como una empresa pública. Con la presión de no generar utilidades para pagar sus deudas, esta debía ser vendida. Pero la venta completa no era significativa para el mercado. Se privilegiaba el componente de telecomunicaciones, seguido de la distribución y comercialización de energía.

El tercer aspecto de la desinstitucionalización estuvo vinculado con las rebeliones culturales acontecidas en diferentes lugares del mundo, siendo Cali lugar de una de estas manifestaciones. Debido a que el Estado es percibido como una institución que "adolece de múltiples enfermedades y perdió definitivamente su rumbo norte y su identidad", la generación de la estabilidad normativa se ha desvanecido en el aire. Además de los problemas y conflictos sociales que padece, "sufre de una pandemia grave como es la corrupción y la tramitomanía" (Gil, s.f.). La agudización de los conflictos en los primeros años de "la intervención" incluyó a otros actores y nuevos intereses que dieron origen a las tomas por parte de los trabajadores en los años de 2001-2002 y 2004.

En un mensaje alcanzo a ver que Luis Hernández escribe que se diga que nos encerramos en el CAM. Mi preocupación era que no estaba preparado ni había traído nada, sin embargo, me dispongo a enviar el mensaje a todos los sindicatos y estar al pendiente de una respuesta, todo dentro de una oficina. En horas de la tarde que decidí

\footnotetext{
${ }^{12}$ Si bien el Estado de Bienestar en Colombia nunca se ha desarrollado siguiendo una de las tipologías construidas para los países del primer mundo (Pico, 1987; Esping-Andersen 1993; Cohen, 2007), esto no significa que su crisis lo haya llevado a su desaparición. Inversiones sociales en educación, vivienda, salud, pensiones de jubilación, empleo en la burocracia oficial, subsidios de desempleo, de invalidez, transporte público módico, cuidado de los niños, reparación de las víctimas, atención de los desplazados, entre otros, han ido creciendo. Las utilidades financieras que una empresa pública del orden municipal genera, permiten aumentar la inversión social de la ciudad, como ocurrió con Emcali desde 1960 hasta mediados de los noventa.
}

Varón, D., (2020). Hacia una integración de la antropología urbana, del trabajo y las organizaciones. Reflexiones entre empresa y ciudad en Cali - Colombia. Jangwa Pana, 19 (1). Doi: https://doi.org/10.21676/16574923.3756 
bajar de la oficina veo cadenas, todo cerrado y algo de comida, mi pensar era que había una forma muy desorganizada con la partición del tema. En todo esto hubo una mínima organización (...) yo era el encargado de todo el tema internacional, de traducción y el interactuar con los sindicatos para pedir todo el apoyo, solidaridad y pronunciamientos donde solicitaba a los sindicatos pronunciarse al gobierno nacional en ese mismo instante. (Entrevista 1, agosto 20 de 2014)

Aunque se puedan cuestionar las organizaciones de las tomas, el derecho a la vida fue vulnerado también en Emcali, alcanzando la cifra récord de 70 actos de violencia entre los que se sumaron atentados, hostigamientos, homicidios y desplazamiento forzado, en el periodo de 1996 y 2003. Pero estos niveles extremos de violencia no eran sino el reflejo del proceso de desinstitucionalización vivido en el país por las luchas entre carteles del narcotráfico, la delincuencia armada y las acciones de grupos al margen de la ley. Una vez intervenida Emcali, las acciones violentas no pararon (Valencia y Celis, 2012; Varón, 2018b).

Parte de la alta rotación de directivos se debió a amenazas contra la vida, al oponerse a un estilo u otro de gestión. Algunos visos de control se pudieron asomar después de la tercera toma, en la que la jefatura de seguridad adaptó tecnologías para generar un control interno (Entrevista 3, febrero 6 de 2015; González, 2016). El autocontrol no había alcanzado los niveles de civilización exigidos para salir de la desinstitucionalización. La paradoja radicó en que la jefatura de seguridad terminó vinculada en el proceso adelantado por la operación dragón y el caso de Indumil. Las recientes sentencias judiciales han encontrado responsabilidad de exmilitares y exparamilitares con la muerte de sindicalistas, campesinos y líderes de la sociedad civil ${ }^{13}$.

Finalmente, el neoliberalismo había trastornado "los viejos marcos institucionales y burocráticos", introduciendo nuevos modos de gestión en el contexto laboral. Es decir que el cuarto elemento de desinstitucionalización estuvo representado por los contratos flexibles que incidieron en el sentido de identidad institucional. En el período de intervención, la "nómina paralela" vía la subcontratación aumentó significativamente. Los contratos laborales ofrecidos por las empresas temporales aumentaron en número, pero incidieron en la disminución del salario pagado al trabajador y, desde luego, en la calidad del servicio prestado. Desaparecieron los procesos de inducción y, con este, el sello distintivo de la identidad institucional (Entrevista 4, agosto de 2015). El usuario no distinguió la diferencia entre el trabajador oficial y el contratista, de forma que el posicionamiento de la empresa fue cediendo ante la competencia que prestó el servicio con más eficiencia, eficacia y efectividad.

\footnotetext{
13 En enero del año 2019 los medios de comunicación informaron que el juzgado cuarto especializado de Cali había condenado a cuatro años de prisión a tres oficiales retirados del Ejército, señalados de elaborar un minucioso plan para atentar contra la vida del senador Alexander López, de varios dirigentes sindicales de Emcali y de la defensora de Derechos Humanos Berenice Celeita. La denominada “Operación Dragón” se adelantó en el año 2004 y, según el senador Alexander López, fue contratada por la entonces superintendente de servicios públicos y hoy funcionaria del gobierno Duque, por lo que elevarán a instancias internacionales la decisión judicial para que continúen las investigaciones. https://www.wradio.com.co/noticias/regionales/condenados-militares-r-por-plan-para-asesinar-a-lideres-de-ddhh-ypoliticos-en--valle/20190123/nota/3854142.aspx (consultado el 1 de octubre de 2019).
}

Varón, D., (2020). Hacia una integración de la antropología urbana, del trabajo y las organizaciones. Reflexiones entre empresa y ciudad en Cali - Colombia. Jangwa Pana, 19 (1). Doi: https://doi.org/10.21676/16574923.3756 
El pésimo servicio prestado por las Empresas Municipales de Cali incidió en la disminución de productividad, en la reducción de usuarios e indiscutiblemente de los ingresos. La baja inversión para la actualización tecnológica perduró durante varios años de la intervención. Las utilidades disminuidas estuvieron orientadas al pago de la deuda, a los servicios financieros que fueron negociados durante el "todos ponen". Para el año 2007 la salud financiera de Emcali había salido de urgencias. En el año 2010, los directivos de la SSPD habían anunciado la devolución de Emcali a la administración del municipio de Cali, asunto que se postergó por tres años.

Durante los trece años de intervención de las Empresas Municipales de Cali, los alcaldes pudieron desarrollar la ciudad mediante otros proyectos como el Sistema Integrado de Occidente (MIO), las mega obras, Ciudad Paraíso, etc. Durante las últimas décadas "se produjeron desviaciones de recursos generando desigualdad en términos redistributivos y restringiendo las posibilidades de los ciudadanos para alcanzar niveles de vida superiores, al impedir que el uso de los recursos públicos sea óptimo en beneficio de la comunidad" (Martínez y Ramírez, 2005, p. 51).

En los tres últimos períodos de los alcaldes se ha empezado a proyectar la ciudad con un gran desafío en materia de infraestructura urbana, a fin de aumentar la movilidad entre los municipios que integran el área metropolitana de Cali. El asunto es que Emcali estuvo ausente con sus recursos. Otro de los desafíos sin lugar a duda es la modernización de servicios públicos en una ciudad que sigue creciendo. El hecho de que la nación haya asumido los pagos de la PTAR, le ha permitido a Emcali modernizar la infraestructura del servicio de acueducto y alcantarillado que se ejecuta por estos años en la ciudad. En últimas, Emcali con el proceso de intervención pasó de institución a empresa, lo que le ha permitido estar en los rankings de las 500 empresas más importantes de América Latina en la última década ${ }^{14}$.

\section{Discusión}

La antropología organizacional y los puentes tejidos con la antropología urbana deberán ser capaces de situar el análisis de las ciudades y de sus barrios en procesos más amplios y globales: en sociedades postindustriales. Esto significa pensar el papel de los trabajadores en la construcción del tejido social. Paradójicamente, el capitalismo flexible promueve la pérdida de valores ciudadanos orientados hacia el largo plazo, modificando la solidaridad, la ciudadanía, la lealtad y el compromiso, por ideologías centradas en el presente inmediato.

En esta línea de acción hizo su aparición la "desindicalización”. Una de las estrategias de muchos países ha sido la de satanizar las organizaciones sindicales para realizar procesos de privatización en empresas públicas. Aunque los resultados presentados en el artículo reflejan un estudio de caso, se mantienen a nivel local y se insinúan casos análogos en Colombia. Sin

\footnotetext{
${ }^{14}$ Según el tamaño, Latin Trade en el año 2005 clasificó a las 500 empresas más grandes de América Latina. Emcali ocupó el 445 con ventas netas de 578.0, utilidades netas de 578.0 y activos totales de 2,040.1 (las cifras en millones de dólares). Para el año 2013, se ubicó en el puesto 486 con ingresos de 780,6; ganancias de 156,9 (las cifras en millones de dólares) y el margen de utilidad del 20,1\%. Otros datos citados por el sindicato mayoritario de Emcali, la ubican en el segundo puesto de Colombia. Al respecto no se explica la metodología utilizada para medir su desempeño. HTTP://WWW.VOLTAIRENET.ORG/ARTICLE122755.HTML (Revisado el 4 de mayo de 2017).
}

Varón, D., (2020). Hacia una integración de la antropología urbana, del trabajo y las organizaciones. Reflexiones entre empresa y ciudad en Cali - Colombia. Jangwa Pana, 19 (1). Doi: https://doi.org/10.21676/16574923.3756 
embargo, los resultados parecen extrapolables a otras problemáticas similares, sobre todo en el contexto latinoamericano, donde la importancia de los movimientos sindicales ha sido diezmada y opacada por la violencia extrema, más aún si los gobiernos se plantean ideológicamente de extrema derecha.

El aumento de sindicatos durante los 13 años de intervención fue significativo. Las últimas convenciones colectivas de trabajo fueron firmadas por más de doce manos, representando al igual número de sindicatos. Ante la incertidumbre de una empresa con altas posibilidades de privatización, la relativa seguridad se esperaba al tener fuero sindical. La formación ontológica transmitida por los sindicatos y el reconocimiento del empleador se modificó por la reducción de la autoridad del sindicato entre los trabajadores y las formas de subcontratación (Boltanski y Chiapello, 2002). En este sentido, en Emcali se hizo presente la desformalización y la desinstitucionalización sistemática de las relaciones laborales.

Meses antes de que mataran a Arnulfo, se había creado el Sindicato de Servidores Públicos de Emcali (Serviemcali). Había hecho parte de la junta directiva del sindicato creado para que los profesionales, principalmente ingenieros, se pudieran proteger de las posibles decisiones que trajera la intervención, porque se "venía durísima". (Entrevista 2, noviembre 14 de 2016)

Instituciones tan relevantes como la escuela, la familia y la religión habían depositado la confianza en los mecanismos institucionales para manejar la crisis de la modernidad de la producción. Posteriormente, la responsabilidad de socializar para el trabajo se les asignó a las empresas. Socialización secundaria que en muchas instituciones se había convertido en el sentido de identidad. Con el cambio al libre mercado, la desinstitucionalización se convirtió en un movimiento "profundo", una manera "distinta" de considerar las relaciones entre normas, valores e individuos, lo que significó "un modo absolutamente distinto de concebir la socialización” (Dubet y Martucelli, p. 2000). La desinstitucionalización incidió en la separación de la socialización y la subjetivación, asunto que también se refleja en el trabajo mediante las desigualdades (Dubet, 2006).

La desinstitucionalización es la ausencia del orden moderno y de los procesos reproductores. El rompimiento institucional ya no considera aspectos de prestigio o valoración social trabajar para cierta institución, o de patrones comunes en las formas de socialización. En Emcali se plantea como una mutación, una transformación o una descomposición que ha permitido desplazar el análisis sociológico desde el sistema y la estructura, hacia el actor, con todos sus intereses, poder y la generación de conflictos entorno a la ciudad que todos desean habitar, aunque para ello muchos líderes sindicales perdieron la vida, como el caso de Arnulfo Cárdenas y muchos otros (Varón, 2018b).

El recorrido orientado a establecer la relación empresa-ciudad transita por las lógicas de la ciudadanía. Los conflictos empezados por desacuerdos entre la clase dirigente y empresarial por un modelo organizacional más adecuado para enfrentar la apertura económica de los años 90, también implicaron la continuidad o ruptura de un modelo de ciudad. Además que esta nueva estructura permitía o no la inversión de capital privado, los trabajadores tendrían una

Varón, D., (2020). Hacia una integración de la antropología urbana, del trabajo y las organizaciones. Reflexiones entre empresa y ciudad en Cali - Colombia. Jangwa Pana, 19 (1). Doi: https://doi.org/10.21676/16574923.3756 
participación decisiva con las primeras "tomas" del edificio administrativo ubicado en el CAM, como se señala en la entrevista 1. Se aumentó la intensidad del conflicto con la participación de diversos grupos integrados por líderes sociales, sindicales, empresarios, directivos de la empresa, partidos políticos de la región y el país, e incluso entidades internacionales como la Organización Internacional del Trabajo.

\section{Conclusiones}

Realizar una investigación que integre las interrelaciones entre ciudad y empresa requiere un esfuerzo enorme por prestar atención a las conexiones de largo plazo, rompiendo con los enfoques que se centran en el aquí y ahora. Se ha tomado como ejemplo una organización que presta servicios públicos domiciliarios en el suroccidente de Colombia y su representatividad implica los años de permanencia en el tiempo y su compromiso social defendido por las organizaciones sindicales: muchos de sus líderes han sido amenazados y asesinados. Emcali cuenta con una de las infraestructuras más grandes del país: 3000 hectáreas de cuencas hidrográficas, cuatro plantas de producción de agua potable, $2600 \mathrm{~km}$ de acueducto, dos plantas de tratamiento de aguas residuales, 30 centrales telefónicas, 527 $\mathrm{km}$ de fibra óptica y 18 subestaciones de energía ${ }^{15}$. En la edición $\mathrm{N}^{\circ} 1826$ de la revista Semana ${ }^{16}$, fue ubicada en el puesto 57 de las 100 empresas más grandes de Colombia durante el año 2016. Su ingreso operacional fue de 1798 598, activos por 6596 875, pasivos de 3 331730 y patrimonio de 3265145 (las cifras se presentan en millones de pesos). El año 2016 fue agridulce para las empresas que prestan servicio de energía. Mientras que Electricaribe fue intervenida por la Superintendencia de Servicios Públicos y en la actualidad se han creado dos organizaciones para prestar este servicio en la Costa Atlántica, organizaciones como ISA reportaron utilidades históricas y Celsia incursionó en el sector de energías renovables con una granja solar en el municipio de Yumbo - Valle del Cauca.

Al analizar las interrelaciones entre la antropología urbana y de las organizaciones empresariales y del trabajo se generó una orientación sociohistórica. La distribución del tiempo apeló a la lógica de quien asigna la gerencia general, ya que esto va a tener influencias sobre la gobernanza de la ciudad en materia de infraestructura de los servicios públicos domiciliarios. En los primeros años se generó por acuerdo entre los integrantes del Consejo Municipal (1931 - 1980) y luego, con cambios en los años 90, la asignación se hizo por parte del alcalde. Durante la intervención, fue el gobierno nacional quien nominó el cargo más relevante de Emcali, el cual tuvo distintos matices: en los primeros años, por acuerdo entre el alcalde y el presidente de la República, pero luego de conversaciones subidas de tono se expresó la autonomía del presidente y/o del Superintendente de Servicios Públicos.

Otro de los objetivos propuestos fue desarrollar reflexiones a partir de estas relaciones y los datos producto de la revisión documental, recogidos entre los años 2013 y 2017 en una empresa que presta servicios públicos, ubicada en Cali - Colombia. En efecto se puede

\footnotetext{
${ }^{15}$ Corporación de Servicios Jurídicos Profesionales Comunitarios SEMBRAR. Colombia: violencia y derechos humanos. Revisado el 1 de mayo de 2017 https://studylib.es/doc/1093956/pdf---3.5-mb

${ }^{16}$ Esta edición especial incluyó información de Colombia y del mundo entre el 30 de abril y mayo del año 2017. Señala que para el año 2015, Emcali ocupó el puesto 54. En comparación con el comportamiento del sector, Emcali soporta un lastre pos su pasivo pensional y altos costos laborales.
}

Varón, D., (2020). Hacia una integración de la antropología urbana, del trabajo y las organizaciones. Reflexiones entre empresa y ciudad en Cali - Colombia. Jangwa Pana, 19 (1). Doi: https://doi.org/10.21676/16574923.3756 
concluir que, en el año 2013, fue el alcalde Rodrigo Guerrero quien recibió de manos de Juan Manuel Santos la administración de Emcali. El alcalde señaló que el proceso de selección requería tiempo, debido a que se requería "el gerente de una ciudad". No es extraño que se eligiera como Gerente General de Emcali al director de Planeación Municipal. Óscar Pardo, de formación como ingeniero civil, con experiencia como docente, investigador y directivo, estaría a cargo de las decisiones de la empresa más grande de servicios públicos del suroccidente colombiano. En su cargo anterior había dejado listo el Plan de Ordenamiento Territorial, ese que otro director debería entrar a ejecutar.

Durante el período de intervención, los recursos financieros fueron invertidos en pagar las deudas adquiridas años atrás por Emcali, de forma que la ciudad dejó de recibir renta e inversión social. Los deseos de sanear las finanzas y generar las utilidades necesarias para aportar al crecimiento de la ciudad sigue siendo el deseo de muchos dirigentes locales. La revisión de fuentes secundarias permitió comprender las nuevas dinámicas de la empresa. Sin embargo, estos estudios de caso deberían ser complementados con casos de estudio que centren su atención en el vínculo empresa-ciudad, y no solamente concentrarse en el desarrollo de eficiencia empresarial, descuidando las interrelaciones con el entorno sociocultural.

Esta miopía de algunas disciplinas opta por comprender las relaciones internas objeto de su ambiente laboral y por el monitoreo de las variables del entorno para tomar decisiones de acuerdo con los cambios de su macroentorno. Al dar prioridad a la prospectiva, se descuidan los estudios sociohistóricos. Se pierde la riqueza producto de la retrospectiva. En este sentido, la antropología tiene herramientas conceptuales, metodológicas y teóricas para aumentar su comprensión sobre las interrelaciones entre empresas y ciudades, y los significados que tanto ciudadanos como trabajadores tienen de sus "patrimonios industriales".

\section{Agradecimientos}

El autor agradece al Dr. Carlos Alberto Patiño Villa, profesor titular de la Universidad Nacional de Colombia y director del Instituto de Estudios Urbanos, por los comentarios realizados tanto en el proceso de evaluación como durante la disertación de tesis realizada en mayo de 2018.

\section{Referencias bibliográficas}

Almario, Ó. (2013). La configuración moderna del Valle del Cauca, 1850 - 1940. Espacio, poblamiento, poder y cultura. Popayán: Editorial Universidad del Cauca.

Arturo, J. (1993). Estudios contemporáneos de cultura y Antropología Urbana. Maguaré, 9, 53-72.

Babor, S. (2009). Un antropólogo en la empresa. De la cultura de la gestión a la gestión de la cultura. Buenos Aires: Gran Aldea Editores.

Boiral, O. (2005) Las normas Iso 9000: Del imperativo comercial al "rito de paso" organizacional, en Nuevo Pensamiento Administrativo. Cali, Univalle.

Boltanski, L. y Chiapello, É. (2002). El nuevo espíritu del capitalismo. Madrid: Ediciones Akal.

Varón, D., (2020). Hacia una integración de la antropología urbana, del trabajo y las organizaciones. Reflexiones entre empresa y 
Bonilla, E. y Rodríguez, P. (2005). Más allá del dilema de los métodos. La investigación en ciencias sociales. Bogotá: Grupo editorial norma - Universidad de los Andes.

Camacho, M. (2005). Las empresas públicas de Santiago de Cali en el siglo XX. Informe final. Cali: Universidad del Valle (Texto inédito).

(2006). Emcali en la historia de Cali. Cali: Empresas Municipales de Cali.

(2007). La encrucijada de los servicios públicos en Cali (1961 - 2004). Cali: Secretaria de Cultura y Turismo del Valle del Cauca.

Cardona, A. (2002). Evaluación integral a la Gestión Fiscal de EMCALI E.I.C.E. E.S.P. Vigencia 2001. Contraloría Municipal de Santiago de Cali. Mayo de 2002.

CIDSE (1994a) “Alcantarillado". Retrospectiva urbana y servicios públicos en Cali 1900 1993. Cali: Centro de Investigación de la Facultad de Ciencias Sociales y Económicas de la Universidad del Valle (Cidse) Cali: Emcali.

(1994b) "Descripción histórica del desarrollo de Acueducto en Cali". Retrospectiva urbana y servicios públicos en Cali 1900 - 1993. Cali: Centro de Investigación de la Facultad de Ciencias Sociales y Económicas de la Universidad del Valle (Cidse) Cali: Emcali.

(1994c) "Descripción histórica del desarrollo de energía eléctrica en la ciudad de Cali". Retrospectiva urbana y servicios públicos en Cali 1900 - 1993. Cali: Centro de Investigación de la Facultad de Ciencias Sociales y Económicas de la Universidad del Valle (Cidse) Cali: Emcali.

(1994d) "Servicio telefónico". En: Retrospectiva urbana y servicios públicos en Cali 1900 1993. Cali: Centro de Investigación de la Facultad de Ciencias Sociales y Económicas de la Universidad del Valle (Cidse) Cali: Emcali.

(1994e) Retrospectiva urbana y servicios públicos en Cali 1900 - 1993. Cali: Centro de Investigación de la Facultad de Ciencias Sociales y Económicas de la Universidad del Valle (Cidse) Cali: Emcali.

Celis, J. (2004). Sindicatos y territorios. Dimensiones territoriales de la acción sindical. Aproximación teórica y descripción de experiencias colombianas. Medellín: Escuela Nacional Sindical.

Collins, R. (1996). Cuatro tradiciones sociológicas. México: Universidad Autónoma Metropolitana de Iztapalapa.

Coriat, B. (2005). El taller y el cronómetro. Ensayos sobre el taylorismo, el fordismo y la producción en masa. México: Editorial Siglo XXI.

Dávila, C. (2000). Cultura organizacional: ¿Un instrumento de la gerencia? Algunas consideraciones para su estudio. Innovación y cultura de las organizaciones en tres regiones de Colombia. Bogotá: Corporación Calidad.

De La Pradelle, M. (2000). La ville des anthropologues. T. Paquot, M. Lussault, S. BodyGendrot, La ville et l'urbain l'état des savoirs. París: Éditions la Découverte, 45-52.

Deegan, M. (2012). Sociólogas pioneras y la Sociedad Sociológica Americana: patrones de exclusión y participación. CS 10, julio-diciembre, 313-338.

Dubet, F. (2006). Injustices. L'expérience des inégalités au travail. Paris: SEUIL Edición.

Dubet, F. y Martuccelli, D. (2000). ¿En qué sociedad vivimos? Buenos Aires: Editorial Lozada.

Esteva Fabregat, C. (1984). Antropología industrial. Barcelona: Anthropos.

Varón, D., (2020). Hacia una integración de la antropología urbana, del trabajo y las organizaciones. Reflexiones entre empresa y 
EMCALI - Empresas Municipales de Cali (1961) La ciudad de Cali y el empréstito BID9TF. Cali: Empresas Municipales de Cali.

Gaceta Municipal N 747. Cali: Archivo Histórico de Cali. Mayo de 1961 a enero de 1962.

García, B. (2008). De la antropología a la antropología urbana. Gazeta de Antropología, 24 (2), artículo 50, http://hdl.handle.net/10481/6955

García, C. M. (2006). Una aproximación al concepto de cultura organizacional. Universitas Psychologica, 5 (1), 163-174.

García, C. (2009) La cultura organizacional como una forma de control social. Psicología del trabajo y de las organizaciones. Reflexiones y experiencias de investigación. Bogotá: Universidad Santo Tomás, 275 - 295.

Gil, H. (s.f.) Desinstitucionalización de las organizaciones. http://www.gerencie.com/desinstitucionalizacion-de-las-organizaciones.html

(Visitado el 10 de mayo de 2016).

González, S. (2003). El derrumbe de Emcali, golpe mortal a los caleños. El derrumbe de Cali. Cali: Freddy León Cuellar, 123-209.

González, Y. (2016). Análisis del conflicto laboral entre el sindicato de trabajadores de EMCALI - SINTRAEMCALI y EMCALI EICE ESP en el periodo 2004-2012 (Caso 2356 OIT). Cali: Universidad del Valle. Facultad de Ciencias de la Administración.

Guerreros, J. (2005). Antropología Urbana. Un recorrido histórico y teórico. Textos Antropológicos, Volumen 15, Número 1, pp. 137-144.

Guzmán, Á. (1993). Diagnóstico sobre la violencia homicida en Cali, 1993, Cuaderno de Trabajo CIDSE $N^{\circ}$ 20, Cali: Universidad del Valle, 1-53.

(2012) Ciudad y violencia: Cali en el siglo XX. Moreno, Esteban (Coord). Historia de Cali, Siglo XX. Tomo II Política. Cali: Universidad del Valle, pp.327-373.

Hammersley, M. y Atkinson, P. (2001). Etnografía. Métodos de investigación. Barcelona: Ediciones Paidós.

Harvey, D. (2013). Ciudades rebeldes Del derecho de la ciudad a la revolución urbana. Madrid. Ediciones Akal.

Homobono, J. (2000). Antropología urbana: itinerarios teóricos, tradiciones nacionales y ámbitos temáticos en la exploración de lo urbano. Cuaderno de AntropologíaEtnología, núm. 19, Donostia, pp.15-50.

Lacarrieu, M. (2007). Una antropología de las ciudades y la ciudad de los antropólogos. Nueva Antropología: La antropología en cuestión: cinco ensayos temáticos y un estudio de caso, 67, 13-40.

Lacarrieu, M.; Carman, M. y Girola, M. (2009). Miradas antropológicas de la ciudad: desafíos y nuevos problemas. Cuadernos de Antropología Social, 30, 2009, 7-16.

Mairal, G. (2001). Antropología de las ciudades históricas. Revista de Antropología Social $10,9-15$.

Martínez, E. (2014). Configuración urbana, habitar y apropiación del espacio. Barcelona: XIII Coloquio Internacional de Geocrítica. El control del espacio y los espacios de control 5-10 de mayo, 1-21.

Martínez, E. \& Ramírez, J. (2005). La corrupción y los costos de transacción. Una mirada desde la contratación estatal colombiana. Cuadernos de Administración, 33, 37-59.

Martínez, P. \& Buitrago, Ó. (2011). Cali: una metrópoli regional en movimiento. La Planeación Municipal y los Procesos de Metropolización. Cali: Universidad del Valle.

Varón, D., (2020). Hacia una integración de la antropología urbana, del trabajo y las organizaciones. Reflexiones entre empresa y 
Ochoa, H. (2010). Modelo de estructura empresarial para las Empresas de Servicios Públicos: el caso de Emcali. Estudios Gerenciales, 59, 9-22.

Pesqueux, Y. (2009). Institución y Organización. Cuadernos de Administración, 41, 7-25. Picó, J. \& Serra, I. (2010). La escuela de Chicago. Madrid: Siglo Veintinuo Editores.

Pineda, R. (1993). Grandes temas de la Antropología Urbana. Maguaré, 9, 9-42.

Prieto, E. Y Patiño, C. (2017) Introducción. Construcción de Metrópolis. Identificación de proceso de planeación para un área metropolitana eficiente. Bogotá: Universidad Nacional de Colombia. Instituto de Estudios Urbanos. Editorial Planeta.

Rempel, W. (2011). En la boca del lobo. La historia jamás contada del hombre que hizo caer al cartel de Cali. México D.F.: Grijalbo.

Rocca, J. (1998). Antropología industrial y de la empresa. Barcelona: Editorial Ariel.

(2001). ¿antropólogos en la empresa?: A propósito de la (mal)llamada cultura de empresa. Etnográfica, V (1), 69-99.

Sáenz, J. (2010). Élite política y constructores de ciudad. Cali 1958 - 1998. Serie Humanidades, Derecho y Ciencias Sociales. Cali: Universidad Icesi.

Salcedo, A. \& Zeiderman, A. (2008). Antropología y Ciudad: Hacia un análisis crítico e Histórico. Antípoda 7, julio-diciembre, 63-97.

Trujillo, J. (2010). Antropología en México y España: industria, trabajo y organizaciones. Antropol.sociol 12, Enero - Diciembre, 197-226.

Urrea, F. (2011). La conformación paulatina de clases medias negras en Cali y Bogotá a lo largo del siglo XX y la primera década del XXI. Revista de Estudios Sociales, 39, 24 41.

(2012). Transformaciones sociodemográficas y grupos socio-raciales en Cali, siglo XX e inicios del siglo XXI. Garzón, José (Coord.). Historia de Cali, Siglo XX. Tomo I Espacio Urbano. Cali: Universidad del Valle, 145-194.

Urrea, F. y Celis, J. (2016). Los estudios laborales en Colombia entre 1993 y 2014. Enrique de la Garza (Ed.), Los estudios laborales en América Latina. Orígenes, desarrollo y perspectivas. México, Universidad Autónoma Metropolitana - Iztapalapa y Anthropos Editorial, 127-156.

Valencia, L. \& Celis J. (2012). Sindicalismo asesinado. Reveladora investigación sobre la guerra contra los sindicalistas colombianos. Bogotá: Editorial Debate.

Varela, E. (2002). Cómo evitar el colapso de Emcali. Los retos de la provisión adecuada de servicios públicos domiciliarios en Colombia. Cali: Universidad del Valle. Facultad de Ciencias de la Administración.

(2007). Gestión y Gobernabilidad en Emcali. Liberalización de los servicios públicos y vulnerabilidad de las burocracias. Bogotá: Ecoe Ediciones.

(2008). Burocratización y modos de gestión en los servicios públicos. Bogotá: Ecoe Ediciones.

Varón, D. (2018a). El proceso de institucionalización de las Empresas Municipales de Cali - Colombia (1931 - 2016). Relaciones de poder y conflicto durante su intervención. Medellín: Universidad Eafit.

(2018b). Las violencias en las Empresas Municipales de Cali - Colombia entre 1995 y 2013. Paramilitares se atribuyeron su muerte. Revista Latinoamericana de Antropología del Trabajo, 2 (3), 1- 24. ISSN 2591-2755. 
Vásquez, E. (1990). "Historia del desarrollo urbano en Cali”. Boletín Socioeconómico, 20, $1-28$.

(2001). Historia de Cali en el siglo 20. Sociedad, economía, cultura y espacio. Cali: Artes Gráficas del Valle.

(2012) Cali en la primera mitad del Siglo XX: mentalidades y sensibilidad. Gilberto Loaiza et. al. (Eds.) Historia de Cali Siglo XX. Tomo III Cultura. Cali: Programa Editorial Facultad de Humanidades, Universidad del Valle, pp. 28-50.

Vélez, L. (2013). Evolución de las empresas de agua y saneamiento de Medellín y Cali en Colombia: ¿vidas paralelas? Banco Interamericano de Desarrollo. https://publications.iadb.org/bitstream/handle/11319/5946/EMCALI.pdf?sequence=1 (Visitado el 10 de enero de 2018).

Villaveces, S. (1997). Cultura y empresa: La objetivación del Yo para consumir al otro. En, Antropología en la modernidad. Bogotá: Instituto Colombiano de Antropología e Historia (ICANH).

Webber, I. \& Hollingsworth, S. (1975). Orientaciones de valores y modernización en Cali. Webber y Ocampo (comp.) Valores, desarrollo e historia. Popayán, Medellín, Cali y el Valle del Cauca. Bogotá: Ediciones Tercer Mundo.

Whiteford, A. (1963). Popayán y Querétaro. Comparación de sus clases sociales. Bogotá: Universidad Nacional de Colombia.

Zapata, A. y Rodríguez, A. (2008). Gestión de la cultura organizacional. Bases conceptuales para su implementación. Cali: Universidad del Valle.

Zuluaga, B. \& Idrobo, J. (2000). Crisis de la deuda en las Empresas Municipales de Cali y perspectivas. Estudios Gerenciales, 79, $23-45$. 